\title{
Comparative study of acceleration techniques for integrals and series in electromagnetic problems
}

\author{
Noyan Kınayman and M. I. Aksun \\ Department of Electrical and Electronics Engineering, Bilkent University, Bilkent, Ankara, Turkey
}

\begin{abstract}
Most electromagnetic problems can be reduced to either integrating oscillatory integrals or summing up complex series. However, limits of the integrals and the series usually extend to infinity, and, in addition, they may be slowly convergent. Therefore numerically efficient techniques for evaluating the integrals or for calculating the sum of an infinite series have to be used to make the numerical solution feasible and attractive. In the literature there are a wide range of applications of such methods to various EM problems. In this paper our main aim is to critically examine the popular series transformation (acceleration) methods which are used in electromagnetic problems and to introduce a new acceleration technique for integrals involving Bessel functions and sinusoidal functions.
\end{abstract}

\section{Introduction}

Numerical techniques used in the solution of electromagnetic problems require, in general, either evaluating oscillatory integrals over infinite domain or calculating the sums of infinite complex series. For example, the method of moments (MoM) in the spectral domain for two-dimensional geometry requires double-infinite integration of complex highly oscillatory functions; the MoM in the spatial domain employs the spatial domain Green's functions, which are defined as the Hankel transform of the spectral domain Green's function; in the analysis of a periodic structure one needs to employ a periodic Green's function which has double infinite summations; or, in the analysis of a microstrip patch antenna via cavity model, the input impedance or field distribution are written in terms of an infinite sum of modes in the cavity.

If the summations and integrals given in the examples above are evaluated by "brute force" as they appear in the problems, the corresponding methods could be computationally very inefficient, rendering these problems impractical. To overcome this computational burden, special acceleration techniques, also called transformation techniques, for both integrals and summations have been proposed and successfully employed. Since these techniques have been studied for specific problems and compared to only a few other techniques, the potentials of these

Copyright 1995 by the American Geophysical Union.

Paper number 95RS02060.

0048-6604/95/95RS-02060\$08.00 techniques with their advantages and disadvantages have not been examined entirely for electromagnetic problems. Hence the contributions of this paper are in (1) providing the complete set of acceleration techniques used in the electromagnetic problems, (2) comparatively studying the acceleration techniques for integrals and series, and (3) introducing a new acceleration technique for integrals involving Bessel functions and sinusoidal functions. The transformations given and compared in this paper are the Euler transformation [Hildebrand, 1974], Shanks' Transformation [Shanks, 1955; Singh and Singh, 1991a], Wynn's $\varepsilon$ algorithm, the method of averages [Mosig and Gardiol, 1979], the Chebyshev-Toeplitz algorithm [Wimp, 1974; Singh and Singh, 1992b], the $\Theta$ algorithm [Brezinski, 1982; Singh and Singh, 1992a] , the Poisson transformation [Papoulis, 1962], Ewald's transformation [Jordan et al., 1986] , Kummer's transformation, and the method of exponentials.

In section 2, the algorithms of the aforementioned transformation methods are presented, with examples when necessary. Numerical examples and the discussion of the methods are given in section 3 . Finally, in section 4 , conclusions about the transformation methods, with their advantages and disadvantages, are given.

\section{The Transformation Methods}

The principle of a series acceleration method is to transform a slowly convergent sequence, by using a linear or nonlinear mapping, to a new, faster converging sequence. Since there is not any universal algorithm which could work for every type of sequence, one should try differ- 
ent algorithms to obtain the optimum result for the problem under investigation. Hence the acceleration methods used can be divided into two main groups: general methods and specific methods. A general transformation method can be applied to any sequence which can be obtained from an infinite series or an infinite oscillatory integral. Examples of such methods are the Euler transformation, Shanks' transformation, Wynn's $\varepsilon$ algorithm, the Chebyshev-Toeplitz algorithm, and the $\Theta$-algorithm. On the other hand, specific methods are derived by analytically working on the kernel of a series or of an integral. Therefore they can be applied only on their own types, but they usually work better than the general methods. The Method of averages, the Method of exponentials, the Poisson transformation, Ewald's transformation, and Kummer's transformation are the examples. Since the partial sums of an infinite series are used in most of the transformation methods, it would be instructive to define them for an infinite series as follows:

$$
\begin{gathered}
\sum_{i=0}^{\infty} a_{i}=a_{0}+a_{1}+a_{2}+a_{3}+\cdots \\
A_{n}=a_{0}+a_{1}+a_{2}+\cdots+a_{n} \quad n=0,1,2, \cdots
\end{gathered}
$$

where $A_{n}$ denotes the partial sum of (1) for $n$ terms.

\subsection{Euler's Transformation}

Euler's transformation can only be used for an alternating series and the equation for the transformation can be given as [Hildebrand, 1974]

$$
\begin{aligned}
& \sum_{k=0}^{\infty}(-1)^{k} f_{k}= \\
& \quad \frac{1}{2}\left(f_{0}-\frac{1}{2} \Delta f_{0}+\frac{1}{4} \Delta^{2} f_{0}+\cdots+(-1)^{r} 2^{-r} \Delta^{r} f_{0}\right)
\end{aligned}
$$

where

$$
\begin{aligned}
\Delta^{r} f_{k}= & f_{k+r}-\left(\begin{array}{c}
r \\
1
\end{array}\right) f_{k+r-1}+\left(\begin{array}{c}
r \\
2
\end{array}\right) f_{k+r-2} \\
& -\cdots+(-1)^{r-1}\left(\begin{array}{c}
r \\
1
\end{array}\right) f_{k+1}+(-1)^{r} f_{k}
\end{aligned}
$$

The convergence of Euler's transformation can be improved by adding some of the initial terms directly before the transformation. Note that increasing the number of previously added terms could improve the convergence.

\subsection{Shanks' Transformation}

The idea behind Shanks' transformation is that the partial sums of a sequence can be treated as a mathematical transient, and it gives an approximation to the base of the transient which is the result of the infinite summation. The $k$ th order Shanks' transformation, $e_{k}$, is defined in the following form [Shanks, 1955]:

$$
\begin{aligned}
& B_{k, n}=e_{k}\left(A_{n}\right)= \\
& \left|\begin{array}{cccc}
A_{n-k} & \cdots & A_{n-1} & A_{n} \\
\Delta A_{n-k} & \cdots & \Delta A_{n-1} & \Delta A_{n} \\
\Delta A_{n-k+1} & \cdots & \Delta A_{n} & \Delta A_{n+1} \\
\vdots & & \vdots & \vdots \\
\Delta A_{n-1} & \cdots & \cdots & \Delta A_{n+k-1} \\
\hline 1 & \cdots & 1 & 1 \\
\Delta A_{n-k} & \cdots & \Delta A_{n-1} & \Delta A_{n} \\
\Delta A_{n-k+1} & \cdots & \Delta A_{n} & \Delta A_{n+1} \\
\vdots & & \vdots & \vdots \\
\Delta A_{n-1} & \cdots & \cdots & \Delta A_{n+k-1}
\end{array}\right|
\end{aligned}
$$

where $A_{n}$ 's are defined in (2) and $\Delta A_{n}=A_{n+1}-A_{n}$. For a special case, $k=1$, (5) becomes

$$
B_{1, n}=\frac{\left|\begin{array}{cc}
A_{n-1} & A_{n} \\
\Delta A_{n-1} & \Delta A_{n}
\end{array}\right|}{\left|\begin{array}{cc}
1 & 1 \\
\Delta A_{n-1} & \Delta A_{n}
\end{array}\right|}=\frac{A_{n+1} A_{n-1}-A_{n}^{2}}{A_{n+1}+A_{n-1}-2 A_{n}}
$$

and it is known as Aitken's $\delta^{2}$ process [Press et al., 1986]. The iterated Shanks' transformation performs the Shanks' transformation repeatedly until another transformation becomes impossible and is given as [Shanks, 1955]

$$
\begin{array}{rlrl}
B_{k, n} & =e_{k}\left(A_{n}\right), & n \geq k \\
C_{k, n} & =e_{k}\left(B_{k, n}\right), & & n \geq 2 k \\
D_{k, n} & =e_{k}\left(C_{k, n}\right), & & n \geq 3 k
\end{array}
$$

Shanks' transformation (5) is usually applied by increasing the order $k$ continuously until a predefined convergence criterion is satisfied. The evaluation of the determinant in (5) is time consuming for higher orders, and it can be avoided by using the following transformation, called Wynn's $\varepsilon$ algorithm [Brezinski, 1982]:

$$
\begin{aligned}
& \varepsilon_{k+1}^{(n)}=\varepsilon_{k-1}^{(n+1)}+\frac{1}{\varepsilon_{k}^{(n+1)}-\varepsilon_{k}^{(n)}} \quad n, k=0,1, \cdots \\
& \varepsilon_{-1}^{(n)}=0 \quad \varepsilon_{0}^{(n)}=A_{n} \quad n=0,1,2, \cdots \\
& e_{k}(A)=\varepsilon_{2 k}^{(n)}
\end{aligned}
$$

where $n$ and $k$ are the indices for the terms and the order of the transformation, respectively, and only the even order transformations are used; the odd order ones are intermediate values. The importance of Wynn's $\varepsilon$ algorithm 
stems from the fact that the evaluation of the determinant is reduced to a simple iterative equation. On the other hand, the $\varepsilon$ algorithm given in (8), which is called "normal" form, has a disadvantage in that it may suffer from cancelation errors due to the computers arithmetic in the case of $\varepsilon_{k}^{(n+1)} \cong \varepsilon_{k}^{(n)}$. The cancelation errors occur because there is a subtraction process in the denominator of (8), and they can be avoided to some extent by using the progressive or particular rules of the algorithm [Brezinski, 1991].

\subsection{Method of Averages}

This technique is especially suitable in evaluating the integrals which have a special form of [Mosig and Gardiol, 1979]

$$
I=\int_{a}^{\infty} f(\lambda) \cos (\lambda \rho) d \lambda
$$

where the function $f(\lambda)$ is assumed to have the asymptotic form

$$
\lim _{\lambda \rightarrow \infty} f(\lambda)=C \lambda^{\alpha}
$$

In addition, the partial integrals are defined as

$$
I_{m}^{1}=\int_{a}^{\lambda_{m}} f(\lambda) \cos (\lambda \rho) d \lambda \quad m=1,2, \cdots, M
$$

where $\lambda_{m}$ 's are the successive zeros of the oscillatory function $\cos (\lambda \rho)$. Then the transformation is given by

$I_{m}^{l+1}=\frac{w_{m}^{l} I_{m}^{l}+w_{m+1}^{l} I_{m+1}^{l}}{w_{m}^{l}+w_{m+1}^{l}} \quad \begin{aligned} & l=1,2, \cdots, M-1 \\ & m=1,2, \cdots, M-l\end{aligned}$

where

$$
w_{m}^{l}=\left(\frac{\lambda_{1}}{\lambda_{m}}\right)^{\alpha+1-l}
$$

The value $I_{1}^{M}$ is an approximation to the integral. This method can also be used in the integration of suitable Bessel functions whose asymptotic forms can be expressed as sinusoidal functions.

\subsection{The $\Theta$ Algorithm}

The $\Theta$ Algorithm has been derived from the Wynn's $\varepsilon$ Algorithm, and it can be expressed as [Brezinski, 1982; Singh and Singh, 1992a]

$$
\begin{aligned}
\Theta_{2 k+2}^{(n)} & =\Theta_{2 k}^{(n+1)} \\
& +\frac{\left[\Theta_{2 k}^{(n+2)}-\Theta_{2 k}^{(n+1)}\right]\left[\Theta_{2 k+1}^{(n+2)}-\Theta_{2 k+1}^{(n+1)}\right]}{\left[\Theta_{2 k+1}^{(n+2)}-2 \Theta_{2 k+1}^{(n+1)}+\Theta_{2 k+1}^{(n)}\right]}
\end{aligned}
$$

$$
\begin{aligned}
\Theta_{2 k+1}^{(n)} & =\Theta_{2 k-1}^{(n+1)}+\frac{1}{\Theta_{2 k}^{(n+1)}-\Theta_{2 k}^{(n)}} \quad n, k=0,1, \cdots \\
\Theta_{-1}^{(n)} & =0 \quad \Theta_{0}^{(n)}=A_{n} \quad n=0,1 \cdots
\end{aligned}
$$

where $A_{n}$ 's are the partial sums as defined in (2).

\subsection{The Chebyshev-Toeplitz Algorithm}

The Chebyshev-Toeplitz algorithm requires, first, one to transfer the partial sums to an intermediate series, $t^{(n)}$ 's [Wimp, 1974]:

$$
\begin{gathered}
t_{k+1}^{(n)}=\varepsilon_{k} t_{k}^{(n)}+2 \varepsilon_{k} t_{k}^{(n+1)}-t_{k-1}^{(n)} \quad n, k=0,1 \cdots \\
t_{-1}^{(n)}=0 \quad t_{0}^{(n)}=A_{n}
\end{gathered}
$$

Then the final transformed series is obtained through

$$
T_{k}^{(n)}=\frac{t_{k}^{(n)}}{\sigma_{k}} \quad n, k=0,1,2, \cdots
$$

where

$$
\begin{array}{cc}
\sigma_{k+1}=6 \sigma_{k}-\sigma_{k-1} & k=1,2,3, \cdots \\
\sigma_{0}=1 & \sigma_{1}=3
\end{array}
$$

and

$$
\begin{array}{rlrl}
\varepsilon_{k} & =1 & , & k=0 \\
\varepsilon_{k}=2 & , & k>0
\end{array}
$$

\subsection{The Poisson Transformation}

The main idea behind the Poisson transformation is the reciprocal spreading property of the Fourier transformation; that is, if a function has a narrower support in one domain, it would have a wider support in the other domain. The Poisson transformation can be expressed as [Papoulis, 1962]

$$
\begin{aligned}
& \sum_{n=-\infty}^{\infty} f(t+n T)= \\
& \frac{1}{T} \sum_{n=-\infty}^{\infty} \exp \left(j n w_{0} t\right) F\left(n w_{0}\right) \quad w_{0}=\frac{2 \pi}{T}
\end{aligned}
$$

and can be applied to double series [Lampe et al., 1985]. Here, the transformation of the following doubly infinite series, which appears as the Green's function of twodimensional periodic structure [Michielssen, 1992], is given as an example:

$$
G_{p}=\frac{1}{4 \pi} \sum_{m, n=-\infty}^{\infty} \exp \left(-j \vec{k}_{0} \cdot \vec{\rho}_{m n}\right) \frac{\exp \left(j k_{0} R_{m n}\right)}{R_{m n}}
$$


where

$$
\begin{gathered}
\vec{k}_{0}=k_{x} \hat{x}+k_{y} \hat{y}+k_{z} \hat{z} \\
\vec{\rho}_{m n}=m D_{x} \vec{x}+\left(m D_{x} \tan \Omega+n D_{y}\right) \vec{y} \\
R_{m n}=\sqrt{\left|\vec{\rho}-\vec{\rho}^{\prime}-\vec{\rho}_{m n}\right|^{2}+\left|z-z^{\prime}\right|^{2}}
\end{gathered}
$$

The Poisson transformation of (19) can be found by following the method described by Lampe et al. [1985] as

$$
G_{p}=\sum_{m, n=-\infty}^{\infty} \frac{\exp \left[-j \vec{k}_{\rho}^{m n} \cdot\left(\vec{\rho}-\vec{\rho}^{\prime}\right)-j k_{z}^{m n}\left|z-z^{\prime}\right|\right]}{2 j k_{z}^{m n} D_{x} D_{y}}
$$

where

$$
\begin{gathered}
\vec{k}_{\rho}^{m n}=k_{x}^{m} \hat{x}+k_{y}^{n} \hat{y} \\
k_{x}^{m}=\frac{2 \pi m}{D_{x}}-\frac{2 \pi n}{D_{y} \cot (\Omega)}+k_{x} \\
k_{y}^{n}=\frac{2 \pi n}{D_{y}}+k_{y} \\
k_{z}^{m n}=\sqrt{k_{0}^{2}-k_{\rho}^{m n^{2}}}, \quad k_{0}^{2}>k_{\rho}^{m n 2} \\
k_{z}^{m n}=-j \sqrt{k_{\rho}^{m n^{2}}-k_{0}^{2}}, \quad k_{0}^{2}<k_{\rho}^{m n 2}
\end{gathered}
$$

and $D_{x}$ and $D_{y}$ are the periodicity, and $\Omega$ is the slant angle of the structure. Although (23) converges very fast for $\left|z-z^{\prime}\right| \gg 0$, it converges rather slowly when $\left|z-z^{\prime}\right| \approx$ 0 , as "on-plane" case. To achieve faster convergence in this case, other transformation methods are applied [Michielssen, 1992; Jorgenson and Mittra, 1990], and given in the next two sections.

\subsection{Ewald's Transformation}

This is a very powerful transformation method utilizing the complementary error function [Jordan et al., 1986; Michielssen, 1992] and expressing the series given in (19) as a summation of two different doubly infinite series:

$$
G_{p}=G_{1}+G_{2}
$$

where $G_{1}$ and $G_{2}$ are given by

$$
\begin{gathered}
G_{1}=\frac{1}{8 \pi} \sum_{m, n=-\infty}^{\infty} \frac{\exp \left(-j \vec{k}_{0} \cdot \vec{\rho}_{m n}\right)}{R_{m n}} \\
\sum_{ \pm} \exp \left( \pm j k_{0} R_{m n}\right) \operatorname{erfc}\left(R_{m n} E \pm \frac{j k_{0}}{2 E}\right)
\end{gathered}
$$

$$
\begin{aligned}
& G_{2}=\frac{1}{4 j D_{x} D_{y}} \sum_{m, n=-\infty}^{\infty} \frac{\exp \left[j \vec{k}_{\rho}^{m n} \cdot\left(\vec{\rho}-\vec{\rho}^{\prime}\right)\right]}{k_{z}^{m n}} \\
& \sum_{ \pm} \exp \left( \pm j\left|z-z^{\prime}\right| k_{z}^{m n}\right) \operatorname{erfc}\left(j \frac{k_{z}^{m n}}{2 E} \pm\left|z-z^{\prime}\right| E\right)
\end{aligned}
$$

and $E$ is a constant which has to be selected to allow an approximately equal convergence rate for $G_{1}$ and $G_{2}$. Note that the complementary error function in $G_{1}$ and $G_{2}$ makes these series converge rapidly, which is a consequence of the fact that the complementary error function behaves asymptotically like $\exp \left(-z^{2} / z \sqrt{\pi}\right)$ as $z \rightarrow \infty$ for $|\arg z|<3 \pi / 4$. To compute the complementary error function efficiently, one can use the algorithm given by Gautschi [1970].

\subsection{Kummer's Transformation}

Kummer's transformation makes use of the fact that the rate of convergence of a series is governed by the asymptotic form of that series. Assume that $f_{a}(n)$ is asymptotic to a function $f(n)$; then Kummer's transformation gives [Lampe et al., 1985; Singh et al., 1990]

$$
\begin{aligned}
\sum_{n=-\infty}^{\infty} f(n) & =\sum_{n=-\infty}^{\infty}\left[f(n)-f_{a}(n)\right]+\sum_{n=-\infty}^{\infty} f_{a}(n) \\
& =G_{1}+G_{2}
\end{aligned}
$$

Usually, $f_{a}(n)$ is chosen in such a way that the last series in (29) has a known closed-form expression. However, one can also use the Poisson transformation over the last series if the closed-form expression is not known. Note that the first series in (29) converges rapidly since the asymptotic form is subtracted out. As an example, if Kummer's transformation is applied to the doubly infinite series given in (19), $G_{1}$ and $G_{2}$ are found in the following form:

$$
\begin{aligned}
& G_{1}= \frac{1}{4 \pi} \sum_{m, n=-\infty}^{\infty} \exp \left(-j \overrightarrow{k_{0}} \cdot \vec{\rho}_{m n}\right) . \\
& {\left[\frac{\exp \left(-j k_{0} R_{m n}\right)}{R_{m n}}-\frac{\exp \left(-j k_{0} R_{m n}^{\prime}\right)}{R_{m n}^{\prime}}\right] } \\
& G_{2}= \sum_{m, n=-\infty}^{\infty} \frac{\exp \left[-j \vec{k}_{\rho}^{m n} \cdot\left(\vec{\rho}-\vec{\rho}^{\prime}\right)\right]}{2 j k_{z}^{m n} D_{x} D_{y}} . \\
& \quad \exp \left[-j k_{z}^{m n}\left(\left|z-z^{\prime}\right|+E\right)\right]
\end{aligned}
$$

where

$$
R_{m n}^{\prime}=\sqrt{\left|\vec{\rho}-\vec{\rho}^{\prime}-\vec{\rho}_{m n}\right|+\left(\left|z-z^{\prime}\right|+E\right)^{2}}
$$


and the constant $E$ is chosen as in the Ewald's transformation. Equation (31) can be thought of as the Poisson transformation for the off-plane case. It is also possible to perform additional acceleration on the first series by applying other transformation methods such as Wynn's $\varepsilon$ algorithm [Singh et al., 1990].

\subsection{Method of Exponentials}

This method is primarily used in evaluating the integrals which have the following form [Chow et al., 1991; Aksun, 1995]:

$$
I=\int_{a}^{\infty} x^{n}\left\{\begin{array}{c}
H_{0}^{(2)}(\lambda x) \\
J_{0}(\lambda x) \\
\sin (\lambda x) \\
\cos (\lambda x)
\end{array}\right\} F(x) d x
$$

where $F(x)$ approximated with complex exponentials by using the generalized pencil of function method [Hua and Sarkar, 1989] or Prony's method [Marple, 1987]. Once the function $F(x)$ is approximated by exponentials, the resultant integral will have the following form that can be evaluated analytically:

$$
I=\sum_{i=0}^{N} \alpha_{i} \int_{a}^{\infty} x^{n}\left\{\begin{array}{c}
H_{0}^{(2)}(\lambda x) \\
J_{0}(\lambda x) \\
\sin (\lambda x) \\
\cos (\lambda x)
\end{array}\right\} \exp \left(-\beta_{i} x\right) d x
$$

The integrals involving Bessel and sinusoidal functions arise in a variety of problems in electromagnetics such as the spatial and spectral domain analysis of planarly stratified media via MoM. Although the numerical integration is possible utilizing an appropriate acceleration technique discussed above, the end result will be independent of $\lambda$, implying that for each $\lambda$ one needs to reintegrate (33). This results in relatively large computation time when compared to the analytical evaluation in the method of exponentials for which the parameter $\lambda$ appears explicitly in the resulting expression. As an example, consider the following integral [Aksun, 1995]

$$
G=\frac{1}{4 \pi} \int_{-\infty}^{\infty} H_{0}^{(2)}\left(k_{\rho} \rho\right) \tilde{G}\left(k_{\rho}\right) k_{\rho} d k_{\rho}
$$

where $G$ and $\tilde{G}$ are the Green's functions in the spatial and spectral domain, respectively, and $H_{0}^{(2)}$ is the Hankel function of second kind. The above integral cannot be evaluated analytically directly for the spectral domain Green's functions that are obtained easily for planarly stratified media. Therefore one can take the integral analytically approximating the spectral domain Green's function by complex exponentials. Then the resultant integral can be evaluated using the Sommerfeld identity

$$
\frac{\exp (-j k r)}{r}=-\frac{j}{2} \int_{-\infty}^{\infty} H_{0}^{(2)}\left(k_{\rho} \rho\right) \frac{\exp \left(-j k_{z}|z|\right)}{k_{z}} k_{\rho} d k_{\rho}
$$

\section{Results and Discussion}

In this section, some numerical examples are given and the transformations used here are summarized in Table 1. For all kinds of transformations, relative error is defined as

$$
\zeta_{r}=\left|\frac{\tilde{S}-S}{S}\right|
$$

where $S$ and $\bar{S}$ are the results obtained from direct summation calculated up to sufficient precision and transformation method, respectively. In addition, a convergence criterion is also used to terminate the iterative transformations and is defined as

$$
\text { convergence }=\left|\frac{A_{M}^{k}-A_{N}^{k-1}}{A_{N}^{k}}\right|
$$

where $A_{n}^{k}$ represents the $k$ th level of any iterated transformation, and $A_{N}$ and $A_{M}$ are the last terms of the two consecutive iterations. The summation process is stopped when the convergence is less than a predefined number, $\zeta_{c}$. Note that the convergence checking must be performed several times to ensure proper convergence.

\subsection{Integration Involving Bessel Functions}

Acceleration of an integral involving a Bessel function is considered here as an example for the application of the acceleration techniques discussed above. In the application of the series transformation methods to the integral (39), one has to convert the integral into a sequence, which can be achieved by integrating each cycle of (39) separately and assigning them to the terms of the series.

$$
\int_{0}^{\infty} x J_{1}(x) d x=1
$$

For the purpose of comparison, the results, relative error versus number of terms, obtained from different acceleration methods are given in Figures 1 and 2, and it is observed that the method of averages works better than the other techniques. However, the method of averages requires the asymptotic behavior of the integrand to determine the optimum weights; if the $\alpha$ in (13) is chosen improperly, its convergence could be worse.

The next example is the calculation of the spatial domain Green's functions for the vector and the scalar poten- 
Table 1. Summary of the Transformations Used in the Examples

\begin{tabular}{|c|c|c|}
\hline Abbreviation & Transformation & Brief Explanation \\
\hline Euler & Euler transformation & (3) and (4) are used \\
\hline Shanks 1 & $k$ th-order Shanks' transformation & $\begin{array}{l}\text { The last term (depends on } N \text { ) of the } \\
\text { sequence obtained from (5) }\end{array}$ \\
\hline Shanks 2 & $\begin{array}{l}k \text { th-order iterated Shanks' } \\
\text { transformation }\end{array}$ & $\begin{array}{l}\text { The first term of the last possible } \\
\text { sequence (depends on } N \text { ) } \\
\text { obtained from (7) }\end{array}$ \\
\hline Wynn & Wynn's $\varepsilon$ algorithm & Implemented by using ( 8 ) \\
\hline Averages & Method of averages & Implemented by using (12) and (13) \\
\hline Theta & $\Theta$ algorithm & Implemented by using (14) \\
\hline CT & Chebyshev-Toeplitz algorithm & Implemented by using (15) and (16) \\
\hline Poisson & Poisson transformation & (23) is used \\
\hline Ewald & Ewald's transformation & (27) and (28) are used \\
\hline Kummer & Kummer transformation & (30) and (31) are used \\
\hline Exponentials & method of exponentials & (33) and (34) are used \\
\hline
\end{tabular}

tials for a planarly layered medium whose parameters are $\epsilon_{r}=4.0$ and $t=0.02032 \mathrm{~cm}$ (thickness of the substrate). For this problem the method of averages, found to be the best method for the previous example, is compared to the method of exponentials and the results, the magnitude of the Green's functions versus distance and the CPU time

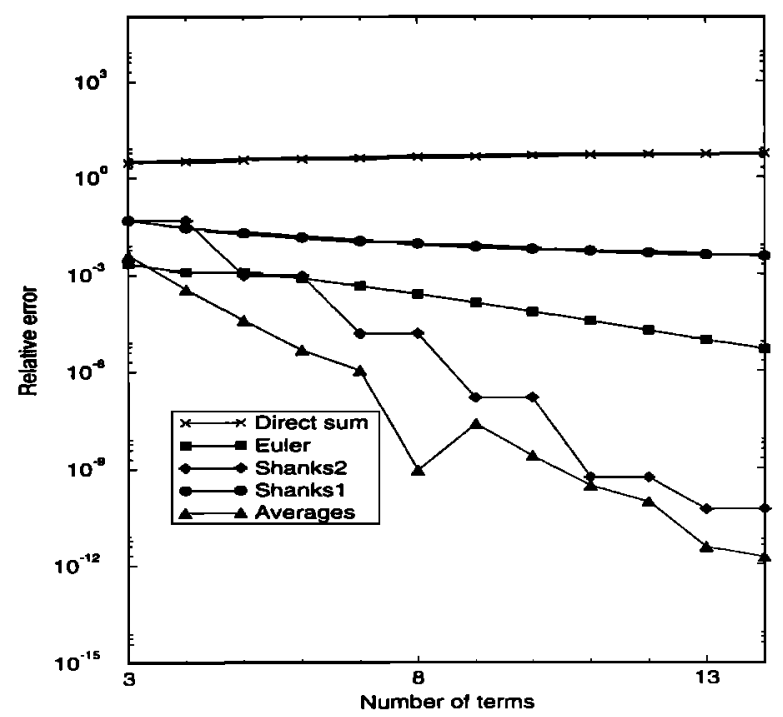

Figure 1. Relative error versus number of terms for the integral (39). measured on a SUN SPARC-5G versus the number of evaluation points $\rho$, are given in Figures 3 and 4, respectively. Since the Green's functions are usually used to find the field components through a convolution integral for a current distribution, it is necessary to evaluate the Green's functions for hundreds of $\rho$ 's. Moreover, the method of

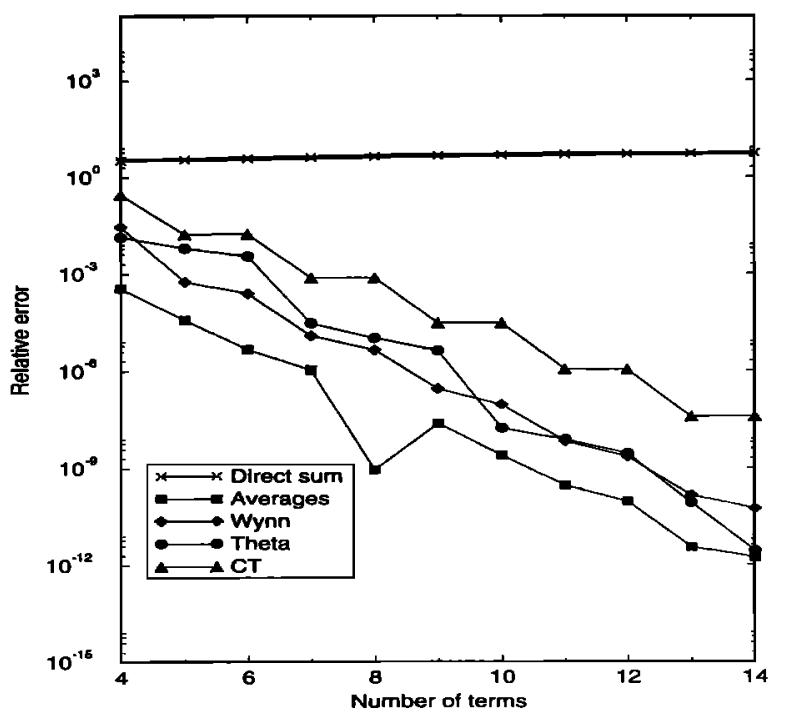

Figure 2. Relative error versus number of terms for the integral (39). 


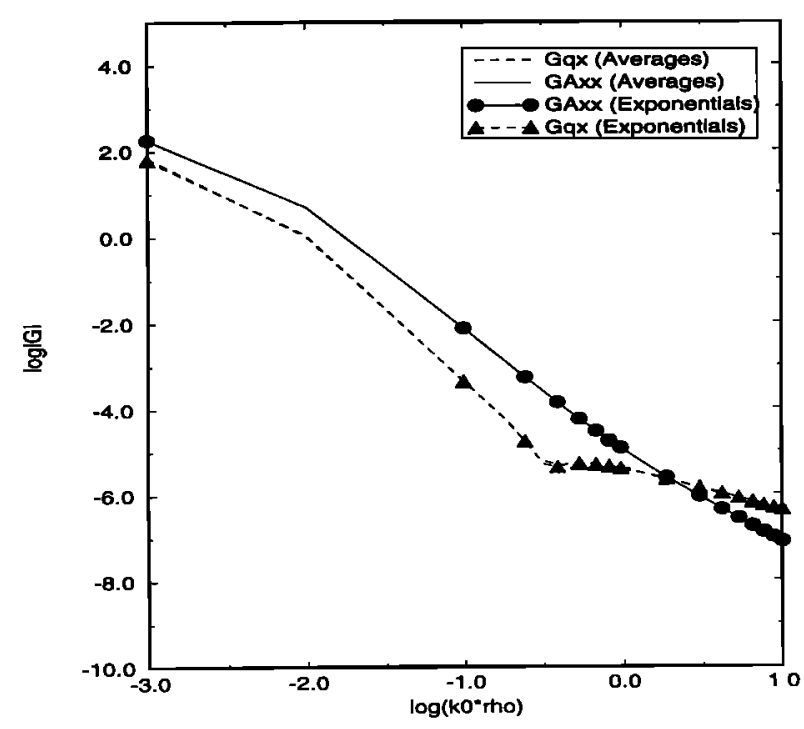

Figure 3. Spatial domain Green's functions evaluated through the method of averages and Method of Exponentials by using (35).

averages requires numerical integration over each cycle of the oscillatory function to compute the partial sums. Consequently, the numerical integration over each cycle must be performed for every different $\rho$ in the application of the method of averages. On the other hand, one needs to approximate the function to be integrated, apart from the kernel, in terms of complex exponentials; then applying

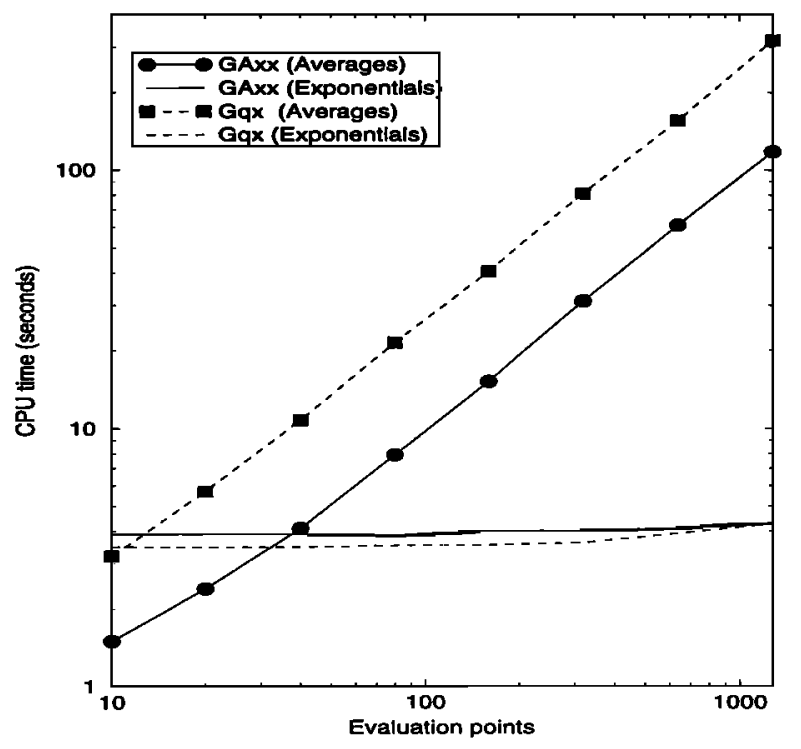

Figure 4. CPU times versus evaluation points for the integral (35). an integral identity results in a function as a function of $\rho$ explicitly. Therefore for each $\rho$ it is enough to evaluate the resulting function. Figure 4 shows that the method of exponentials is faster than the method of averages in case one needs to evaluate the Green's functions over 30-40 points for this specific geometry, and the improvement in the computation time becomes significant as the number of evaluation points increases.

\subsection{Free-Space Periodic Green's Functions}

In this section, acceleration of the infinite summations that appear in the free-space Greens' functions is demonstrated. Assuming a one-dimensional case first, the Green's function for a one-dimensional periodic array of point sources located $d$ units apart in the $z$ direction can be written as [Mosig and Gardiol, 1979; Singh and Singh, 1990]

$$
G=\frac{1}{4 \pi} \sum_{m=-\infty}^{\infty} \frac{\exp \left(-j k R_{m}\right)}{R_{m}}
$$

where

$$
R_{m}=\left[\left(x-x^{\prime}\right)^{2}+\left(y-y^{\prime}\right)^{2}+(z-m d)^{2}\right]^{1 / 2}
$$

The partial sums are obtained for $x=y=0.1 \lambda, z=$ $0.3 \lambda, \lambda=1.0 \mathrm{~m}$, and $d=0.6 \mathrm{~m}$. Figure 5 demonstrates the results, relative error versus number of terms, obtained via some transformation techniques described in section 2.

Another example is the Green's function for onedimensional line sources spaced $d$ units apart along the $x$

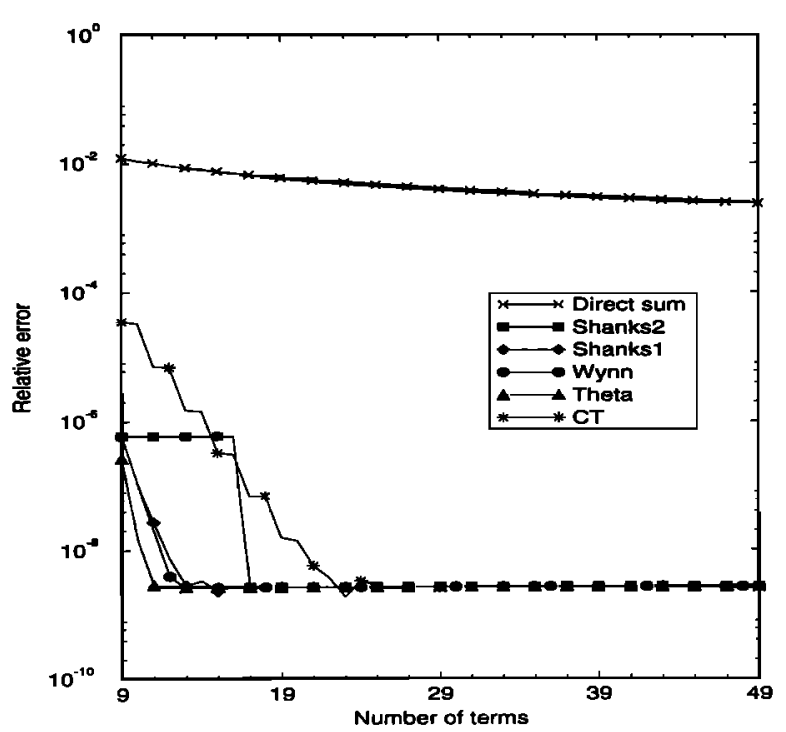

Figure 5. Relative error versus number of terms for the summa. tion (40). 


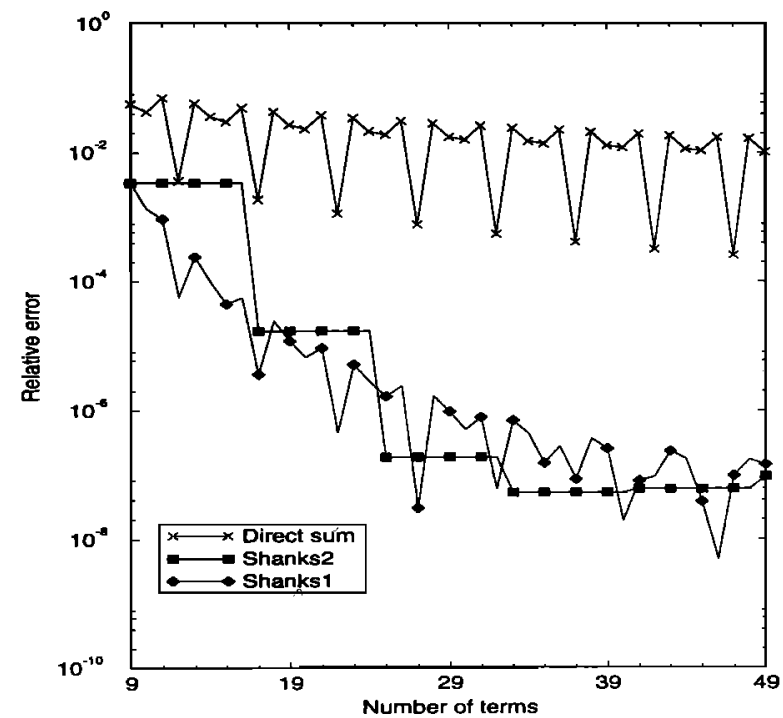

Figure 6. Relative error versus number of terms for the summation (42).

axis with the reference source located at $\left(x^{\prime}, y^{\prime}\right)$

$$
\begin{gathered}
G=\sum_{m=-\infty}^{\infty} \frac{\exp \left[-j\left(k_{x}+2 m \pi / d\right)\left(x-x^{\prime}\right)\right]}{j 2 d k_{y m}} \\
\exp \left(-j k_{y m}\left|y-y^{\prime}\right|\right)
\end{gathered}
$$

where

$$
\begin{gathered}
k_{y m}=\sqrt{k^{2}-\left(k_{x}+2 m \pi / d\right)^{2}}, k^{2}>\left(k_{x}+2 m \pi / d\right)^{2} \\
k_{y m}=\frac{1}{j} \sqrt{\left(k_{x}+2 m \pi / d\right)^{2}-k^{2}}, k^{2}<\left(k_{x}+2 m \pi / d\right)^{2} \\
k_{x}=k \sin (\Theta) \cos (\Phi)
\end{gathered}
$$

and the chosen parameters involved in the series are: $d=$ $0.5 \lambda, x=0.1 \lambda, \lambda=1 \mathrm{~m}, x^{\prime}=y^{\prime}=y=z^{\prime}=0$, and $k_{x}=0$. The results are shown in Figures 6 and 7, and it can be concluded that Wynn's $\varepsilon$ algorithm and Shanks' transformation work better that the other algorithms.

Finally, the free-space periodic Green's function for the two-dimensional case will be investigated. The transformations are performed for a nearly on-plane case for the summation given in (19) in order to have reasonable convergence times for the Kummer and Poisson transformations, which are further accelerated by the Wynn's $\varepsilon$ algorithm. The results are given in Figure 8 for $\lambda_{0}=1.0$ $\mathrm{m}, k_{x}=3, k_{y}=2, D_{x}=D_{y}=0.7 \lambda$, and $z=0.004$, and the numbers near the graphics show the relative errors at the corresponding convergence rate. As a final comment, as $z \rightarrow 0$, the time required for Ewald's transformation for the same convergence rate does not change considerably, whereas the other two methods slow down significantly.

\subsection{Quasi-Dynamic Green's Function}

The quasi-dynamic Green's function for an $x$ directed electric dipole of unit strength located above a microstrip substrate can be written as follows [Chow et al., 1991]:

$$
\begin{aligned}
G_{g 0}= & \frac{1}{4 \pi \varepsilon_{0}}\left[\frac{\exp \left(-j k_{0} r_{0}\right)}{r_{0}}+K \frac{\exp \left(-j k_{0} r_{0}^{\prime \prime}\right)}{r_{0}^{\prime \prime}}\right. \\
& \left.+\sum_{n=1}^{\infty} K^{n-1}\left(K^{2}-1\right) \frac{\exp \left(-j k_{0} r_{n}\right)}{r_{n}}\right]
\end{aligned}
$$

where

$$
\begin{gathered}
r_{0}^{\prime \prime}=\sqrt{\rho^{2}+\left(z+z^{\prime}\right)^{2}} \\
r_{n}=\sqrt{\rho^{2}+\left(z+z^{\prime}+2 n h\right)^{2}}
\end{gathered}
$$

and

$$
K=\frac{1-\varepsilon_{r}}{1+\varepsilon_{r}}
$$

For the evaluation of the summation in (43), the parameters chosen are: $\rho=3 \times 10^{-3} \mathrm{~m}, z=z^{\prime}=0, h=10^{-3}$, $\varepsilon_{r}=12.9$ and $h / \lambda_{0}=0.05$. As pointed out by Chow et al. [1991], for this kind of geometry $G_{q 0}$ needs approximately 80 terms to ensure the desired convergence. However, as shown in Figure 9, the application of the acceleration techniques makes the same series converge in nine terms, which is better than the direct summation corresponding to 80 terms.

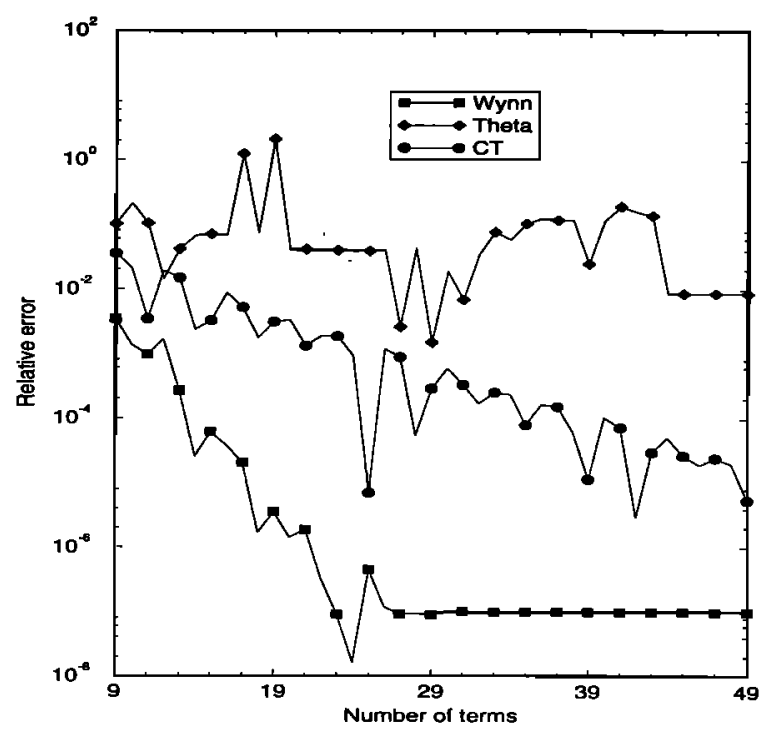

Figure 7. Relative error versus number of terms for the summation (42). 


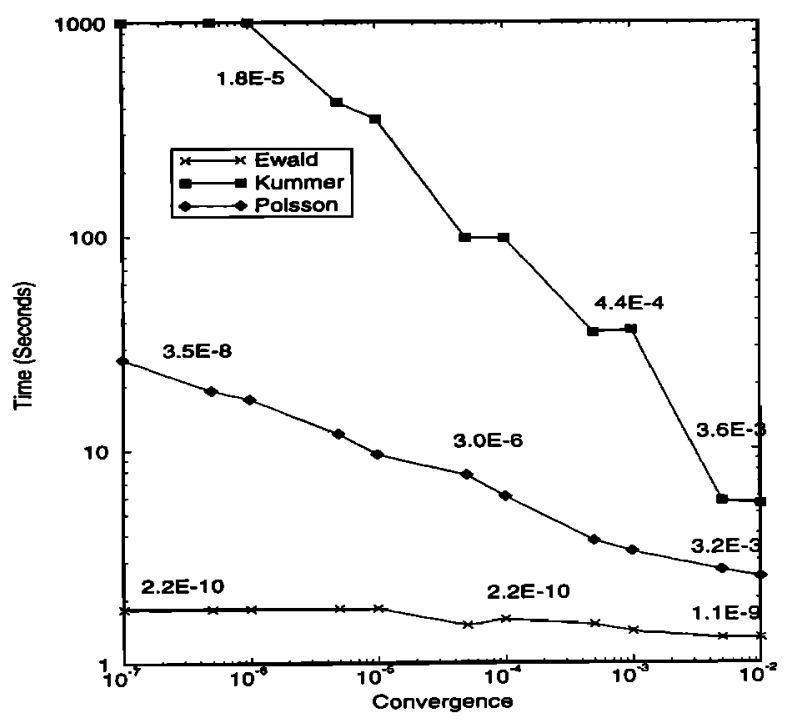

Figure 8. CPU times versus convergence rate $\zeta_{c}$ for different acceleration methods applied to (19).

\section{Conclusion}

Series transformation methods used in electromagnetic problems are studied and compared for some numerical examples in Electromagnetics. Among these transformations, the Euler transformation can only be applied to alternating sequences; even then, other transformation methods may work better than the Euler transformation.

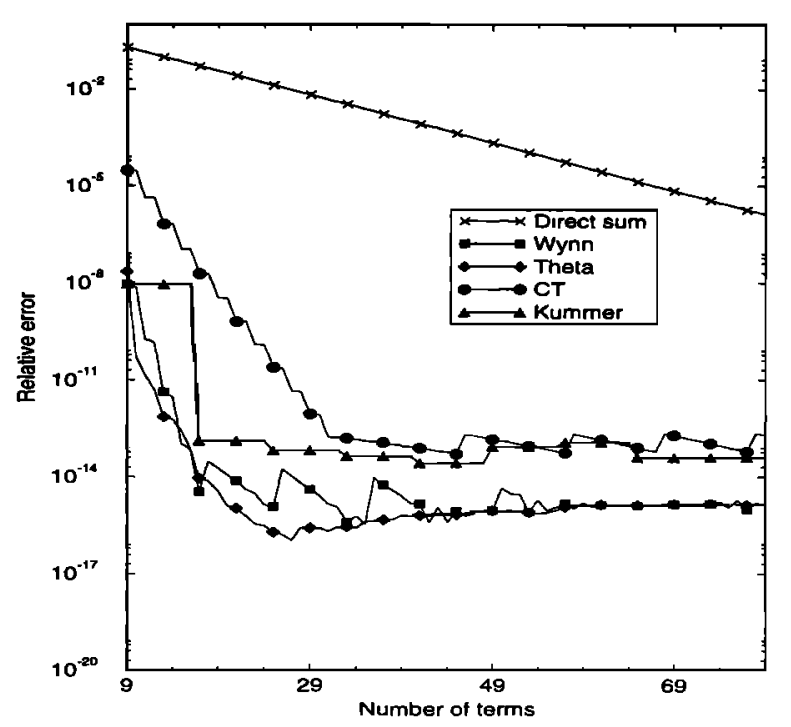

Figure 9. Relative error versus number of terms for the summation (43).
Wynn's $\varepsilon$ algorithm seems to be working better when overall performance is considered and it is prefered over the direct application of higher-order Shanks' transformation because it eliminates the calculation of the determinants in Shanks' transformation. Although there is an exception (see Figure 7), the $\Theta$ algorithm also gives good results in most cases.

On the other hand, the user must be aware of cancelation errors which might occur in the iterative transformations such as Wynn's $\varepsilon$ algorithm. In the case of cancelation errors, the alternate forms of the algorithms should be used [Brezinski, 1991]. In addition, although the $\varepsilon$ algorithm works well for oscillatory sequences, it does not work for monotonic sequences. For monotonic sequences, one can use the $\rho$ algorithm [Singh and Singh, 1991b].

For doubly infinite sums, Ewald's transformation performs much better than the others, and its power stems from expressing the summation in terms of the complementary error functions. There are also general transformation methods on double series, namely Streit's transformation and Haccart's transformation [Brezinski, 1991].

For the integrals involving Bessel and sinusoidal functions, the weighted averages or the method of exponentials can be chosen depending on the application. The method of exponentials is preferable if one needs to evaluate the integral for different values of a parameter because it results in a closed-form expression as an explicit function of the parameter.

Acknowledgments. This work is partially supported by NATO's Scientific Affairs Division in the framework of the Science for Stability program.

\section{References}

Aksun M. I., A robust approach for the derivation of closed-form green's functions, IEEE Trans. Microwave Theory Tech., in press, 1995.

Brezinski, C., Some new convergence acceleration methods, Math. Comput., 39, 133-145, 1982.

Brezinski, C., and R. Zaglia, Extrapolation Methods, Theory and Practice, North-Holland, New York, 1991.

Chow, Y. L., J. J. Yang, D. G. Fang, and G. E. Howard, A closed-form spatial green's function for the thick microstrip substrate, IEEE Trans. Microwave Theory Tech., 39, 588-592, 1991.

Gautschi, W., Efficient computation of the complex error function, SLAM J. Numer. Anal. , 7, 187-198, 1970.

Hildebrand, F. B., Introduction to Numerical Analysis, McGraw-Hill, New York, 1974.

Hua, Y., and T. K. Sarkar, Generalized pencil-of-function method for extracting poles of an EM system from its transient response, IEEE Trans. Antennas Propag., 37, 229-234, 1989.

Jordan, K. E., G. R. Richter, and P. Sheng, An efficient numerical evaluation of the Green's function for the Helmholtz operator on periodic structures, J. Comput. Phys., 63, 222-235, 1986.

Jorgenson, R. E., and R. Mittra, Efficient calculation of the free-space periodic green's function, IEEE Trans. Antennas Propag., 38, 633$642,1990$. 
Lampe, R., P. Klock, and P. Mayes, Integral transforms useful for the accelerated summation of periodic free-space Green's functions, IEEE Trans. Microwave Theory Tech., 33, 734-736, 1985.

Marple, S. L., Digital Spectral Analysis with Applications, PrenticeHall, Englewood Cliffs, N. J., 1987.

Michielssen, E., Electromagnetic plane wave scattering from periodically loaded cylinders and doubly periodic slabs, Ph.D. thesis, Univ. of Ill., Urbana-Champaign, 1992.

Mosig, J. R. and F. E. Gardiol, A dynamical radiation model for microstrip structures, in Advances in Electronics and Electron Physics, edited by P. W. Hawkes et al., vol. 59, pp. 139-233, 1979.

Papoulis, A., The Fourier Integral and Its Applications, McGraw-Hill, New York, 1962.

Press, W. H., B. P. Flannery, S. A. Teukolsky, and W. T. Vetterling, Numerical Recipes, Cambridge Univ. Press, New York, 1986.

Shanks, D., Non-linear transformations of divergent and slowly convergent sequences, J. Math. Phys., 34, 1-42, 1955.

Singh, S. and R. Singh, Application of transforms to accelerate the summation of periodic free-space Green's functions, IEEE Trans. Microwave Theory Tech., 38, 1746-1748, 1990.

Singh, S. and R. Singh, On the use of Shanks' transform to accelerate the summation pf slowly converging series, IEEE Trans. Microwave Theory Tech., 39, 608-610, 1991a.
Singh, S. and R. Singh, on the use of $\rho$ algorithm in series acceleration, IEEE Trans. Antennas Propag., 39, 1514-1517, 1991b.

Singh, S. and R. Singh, A Convergence acceleration procedure for computing slowly convergent series, IEEE Trans. Microwave Theory Tech., 40, 158-171, 1992a.

Singh, S. and R. Singh, On the use of Chebyscher-Toeplitz algorithm in accelerating the numerical convergence of infinite series, IEEE Trans. Microwave Theory Tech., 40, 171-173, 1992b.

Singh, S., W. F. Richards, J. R. Zinecker, and D. R. Wilton, Accelerating the convergence of series representing the free-space periodic Green's function, IEEE Trans. Antennas Propag. , 38, 1958-1962, 1990.

Wimp, J., Toeplitz arrays, linear sequence transformations, and orthogonal polynomials, Numer. Math., 23, 1-17, 1974.

N. Kınayman and M. I. Aksun, Department of Electrical and Electronics Engineering , Bilkent University, Ankara 06533, Turkey. (e-mail: irsadi@ee.bilkent.edu.tr; noyan @ee.bilkent.edu.tr)

(Received March 20, 1995; revised June 30, 1995;

accepted June 30, 1995.) 